\title{
Performance Analysis of Dual-Hop MIMO AF Relaying with Multiple Interferences
}

\author{
Min Li, Min Lin, Wei-Ping Zhu, Senior Member, IEEE, Yongming Huang Member, IEEE, Kai-Kit \\ Wong, Senior Member, IEEE, and Quan Yu
}

\begin{abstract}
In this paper, we investigate the performance of a dual-hop multiple-input multiple-output (MIMO) amplifyand-forward (AF) relay network, where the source, relay and destination are all equipped with multiple antennas. By using maximal-ratio-transmission (MRT) and maximal-ratio combining (MRC) at the transmitter and receiver, respectively, we first obtain the output signal-to-interference-plus-noise ratio (SINR) of the dual-hop AF relay system with multiple co-channel interferences (CCIs) and noise at the relay. Then, we derive exact closed-form expression for the outage probability (OP), and the asymptotic result of average symbol error rate (SER) at high SNR. Finally, computer simulations are provided to validate the performance analysis. Our new analytical expressions not only provide a fast and efficient method to evaluate the system performance, but also enable us to gain valuable insights into the effects of key parameters on the MIMO AF relaying benefit from implementing multiple antennas at each of the three nodes.
\end{abstract}

\section{Index Terms}

amplify-and-forward relaying, multiple-input multiple-output, dual-hop system, co-channel inteferences, outage probability, symbol error rate.

\section{INTRODUCTION}

Multiple-input multiple-output (MIMO) with multiple antennas at both ends of a communication link is considered as a privileged means to enhance the spectral efficiency and improve the reliability of wireless systems [1]. Parallel

This work is supported by the National Natural Science Foundation of China under Grant 61231011 and 61271255, and in part by Jiangsu Province Natural Science Foundation of China under Grant BK20131068.

M. Li and Q. Yu are with the Institute of Communication Engineering, PLA University of Science and Technology, Nanjing 210007, China (e-mail: limin_sclssw@163.com).

M. Lin (corresponding author) is with PLA University of Science and Technology, Nanjing 210007, and also with the National Mobile Communications Research Laboratory, Southeast University, Nanjing 210096, China (e-mail: linmin63@163.com).

W.-P. Zhu is with the Department of Electrical and Computer Engineering, Concordia University, Montreal, QC, Canada H3G 1M8 (e-mail: weiping@ece.concordia.ca).

Y. Huang is with the School of Information Science and Engineering, Southeast University, Nanjing 210096, China (e-mail: huangym@seu.edu.cn).

K. K. Wong is with Department of Electrical and Electronics Engineering, University College London, UK. (e-mail:k. wong@ee.ucl.ac.uk). 
to the well-known MIMO technology, wireless relaying is another efficient approach to improve the performance and extend the coverage of communication networks [2]. Therefore, the huge potential of achieving the benefits of both MIMO technology and relay transmission has attracted a great deal of research interest in the MIMO relaying schemes, which have also been included in the standards for future wireless communication systems [3]. Among the various relaying protocols, amplify-and-forward (AF) is of particular interest due to its simplicity and low implementation cost. When perfect channel state information (CSI) is available, the performance of beamforming (BF) in MIMO AF relaying has been well investigated in the open literatures [4]-[6].

While the aforementioned works have provided valuable insights into the benefits and performance of AF relaying with BF, a key feature of wireless communication systems, namely, co-channel interference (CCI), is unfortunately ignored therein. In practice, CCIs due to frequency reuse often severely degrade the system performance of cellular networks. Therefore, the study on dual-hop AF relay networks with interferences has received significant interest recently. In [7], the authors have derived the outage probability (OP) of a dual-hop fixed-gain AF relaying with interference-limited destination, while the authors of [8] have addressed the scenario of variable-gain protocol and interference-limited relay. Later, the authors of [9] have investigated the performance of fixed-gain dual-hop systems with a Rician interferer. The authors in [10] have investigated the performance of dual-hop CSI-assisted AF relaying systems over Nakagami-m fading channels in the presence of multiple interferers at the relay. A generalized framework for the ergodic capacity analysis of dual-hop fixed-gain AF relaying systems in the presence of interference is presented in [11], where the new expressions for the ergodic capacity are derived over independent but not necessarily identically distributed Nakagami-m fading channels in the presence of a finite number of CCIs. In these works, however, the source, relay and destination are each constrained to a single antenna, and thus the reported analysis results are not applicable to a general case of having multiple antennas as desired in practical network deployments. Under this consideration, based on the assumption of interference-limited system, the authors of [12]-[16] have examined the joint effect of BF and CCI on the dual-hop multi-antenna relaying. More recently, by assuming that the relay is corrupted by a single dominant interferer and noise while the destination is subjected to the noise only, the authors of [17] have analyzed the outage performance of a dual-hop fixed-gain relay system with multiple antennas, which has been extended to both fixed-gain and variable-gain relaying in [18]. In [19], the authors have proposed three relay precoding schemes for a dual-hop system with only relay having multiple antennas, and derived exact outage expressions as well as simple high signal-to-noise ratio (SNR) outage approximations. Furthermore, the ergodic capacity of a dual-hop AF relaying system where the relay is equipped with multiple antennas and subject to CCI and additive white Gaussian noise (AWGN) is analyzed in [20], and the three precoding schemes in [19] have been considered in [20]. The performance analysis of multiuser multiple antenna amplifyand-forward relaying networks employing opportunistic scheduling with feedback delay and CCI over Rayleigh fading channels is presented in [21]. However, it should be pointed out that the common weakness of the prior works is that they limit their analysis to the case where only one of the nodes is equipped with multiple antennas for mathematical tractability. Hence, the joint effects of multiple antennas at each node and multiple CCIs at the relay on the dual-hop AF relaying remain unknown. This observation motivates the work presented in this paper. 
The main objective of this paper is to analyze the performance of a dual-hop MIMO AF relay system in the presence of interferences as well as noise. By considering a general case where each of the source, relay and destination nodes is equipped with multiple antennas and applying the maximum-ratio-transmission (MRT) and maximum-ratio combining (MRC) at the transmitter and receiver, respectively, we first obtain the output signalto-interference-plus-noise ratio (SINR) of the MIMO AF relay system with multiple CCIs and noise at the relay. Then, we derive the exact closed-form OP expression and the asymptotic average SER at high SNR to reveal the array gain and diversity order of the dual-hop variable-gain AF relaying. It is of interest to note that our new analytical results contain part of the work in [4] as a special case when the interferences are ignored. It is also worth-mentioning the differences of our work from the work in [18]. First, by assuming that each node is equipped with a number of antennas, we obtain a unified model of MIMO AF relaying rather than the three scenarios as considered in [18], each corresponding to the case of multiple antennas at one of the three nodes only. Second, the relay is corrupted by multiple interferences in our work, whereas the relay node is subjected to a single dominant interferer in [18]. Thirdly, single integrals are involved for the analytical OP of the variable-gain relaying in [18], but a closed-form OP expression of variable-gain relaying is yielded in our paper. Finally, only OP is investigated in [18], and asymptotic average SER is included in our work. The new analytical expressions can not only provide fast and efficient approaches to evaluate the performance of the system, but also help us to understand the joint effects of key parameters on the performance of the dual-hop AF relaying system, as well as the benefit of implementing multiple antennas.

The rest of the paper is organized as follows. In Section II, we describe the system model, and derive the output SINR of the MIMO AF relay networks with multiple CCIs and noise at the relay. In Section III, we analyze the OP of variable-gain relaying in a closed-form. In Section IV, we derive the asymptotic average SER. Section V provides computer simulations to confirm the validity of the analytical result and examine the key parameters on the dual-hop AF system. Finally, Section VI draws the conclusion of our work in this paper.

Notations: Bold faced letters represent vectors or matrices, $(\cdot)^{H}$ the Hermitian transpose, $(\cdot)^{*}$ the complex conjugate, and $\mathrm{E}[\cdot]$ the expectation. $\exp (\cdot)$ denotes the exponential function, $|\cdot|$ the absolute value, $\|\cdot\|_{F}$ the Frobenius norm. $\aleph_{c}\left(m, \sigma^{2}\right)$ stands for a complex Gaussian distribution with mean $m$ and variance $\sigma^{2}$, and $\min \{a, b\}$ for the minimum of $a$ and $b$. diag $\left(a_{1}, a_{2}, \cdots, a_{N}\right)$ represents an $N \times N$ diagonal matrix with $a_{1}, \quad a_{2}, \cdots, \quad a_{N}$ as its diagonal elements.

\section{SYSTEM MODEL}

We consider a dual-hop MIMO AF relay network with $N_{s}$ antennas at the source $S, N_{r}$ antennas at the relay $R$, and $N_{d}$ antennas at the destination $D$, where the direct link between $S$ and $D$ is unavailable due to severe shadowing. The total communication from $S$ to $D$ via $R$ takes place in two time slots. In the first time slot, the source performs $\mathrm{BF}$ with $\mathbf{w}_{s}$ and transmits the signal $x_{s}(t)$ with $\mathrm{E}\left[\left|x_{s}(t)\right|^{2}\right]=1$ to the relay through the Rayleigh fading channel $\mathbf{H}_{r s}\left(N_{r} \times N_{s}\right)$. Meanwhile, the relay is also corrupted by $N$ CCIs $\left\{x_{i}(t)\right\}_{i=1}^{N}$ obeying $\mathrm{E}\left[\left|x_{i}(t)\right|^{2}\right]=1$. After adopting receive $\mathrm{BF}$ with $\mathbf{w}_{r s}$, the output signal at $R$ can be written as 


$$
y_{r}(t)=\mathbf{w}_{r s}^{H}\left(\mathbf{H}_{r s} \mathbf{w}_{s} \sqrt{P_{s}} x_{s}(t)+\sum_{i=1}^{N} \mathbf{h}_{i} \sqrt{P_{i}} x_{i}(t)+\mathbf{n}_{r}(t)\right)
$$

where $P_{s}$ is the transmit power of the source, $\mathbf{h}_{i}$ the $N_{r} \times 1$ channel vector from the $i$ - th CCI to the relay, and $P_{i}$ the transmit power of the $i$ - th interferer. In the second time slot, the relay first amplifies $y_{r}(t)$ with a gain $G$, and forwards it to the destination through the Rayleigh fading channel matrix $\mathbf{H}_{d r}\left(N_{d} \times N_{r}\right)$. Accordingly, after performing $\mathrm{BF}$, the output signal at $D$ can be written as

$$
y_{d}(t)=\mathbf{w}_{d}^{H}\left(\mathbf{H}_{d r} \mathbf{w}_{d r} G y_{r}(t)+\mathbf{n}_{d}(t)\right)
$$

where $\mathbf{w}_{d r}$ and $\mathbf{w}_{d}$ are the BF weight vector of transmitter at $R$ and that of receiver at $D$, respectively. Without loss of generality, in this paper, we assume that all channels, namely, $\mathbf{H}_{r s}, \mathbf{H}_{d r}$ and $\mathbf{h}_{i}(i=1,2, \ldots, N)$ are subject to Rayleigh fading distribution, whose components are independent and identically distributed (i.i.d.) satisfying $\aleph_{c}(0,1)$. Meanwhile, $\mathbf{n}_{r}(t)$ and $\mathbf{n}_{d}(t)$ are vectors of AWGN with their components obeying $\aleph_{c}\left(0, \sigma_{r}^{2}\right)$ and $\aleph_{c}\left(0, \sigma_{d}^{2}\right)$, respectively.

After some mathematical manipulations, the output SINR at the destination can be written as

$$
\gamma=\frac{P_{s} G^{2}\left|\mathbf{w}_{d}^{H} \mathbf{H}_{d r} \mathbf{w}_{d r}\right|^{2}\left|\mathbf{w}_{r s}^{H} \mathbf{H}_{r s} \mathbf{w}_{s}\right|^{2}}{G^{2}\left|\mathbf{w}_{d}^{H} \mathbf{H}_{d r} \mathbf{w}_{d r}\right|^{2}\left(\sum_{i=1}^{N} P_{i}\left|\mathbf{w}_{r s}^{H} \mathbf{h}_{i}\right|^{2}+\sigma_{r}^{2}\right)+\sigma_{d}^{2}}
$$

It is worth-mentioning that an SINR expression similar to (3) has been obtained in several prior works [4], [5], [18]. However, it was derived only for certain special scenarios, namely, the case of no interference existing [4], the case of no interference and channel errors [5] and the case of only one of the three nodes employing multiple antennas [18]. As such, our work extends the previous system models to a more general case.

By assuming that perfect CSIs are available for both $S-R$ and $R-D$ links at the corresponding node, a MIMO BF scheme with MRT at the transmitter and MRC at the receiver can be applied to enhance the output signal power [4] [5]. Thus, the instantaneous end-to-end SINR at $D$ can be written as

$$
\gamma=\frac{P_{s} G^{2} \lambda_{d r, 1} \lambda_{r s, 1}}{G^{2} \lambda_{d r, 1}\left(\sum_{i=1}^{N} P_{i}\left|\mathbf{u}_{r s, 1}^{H} \mathbf{h}_{i}\right|^{2}+\sigma_{r}^{2}\right)+\sigma_{d}^{2}}
$$

where $\lambda_{r s, 1}$ and $\lambda_{d r, 1}$ are the largest eigenvalues of $\mathbf{H}_{r s}^{H} \mathbf{H}_{r s}$ and $\mathbf{H}_{d r}^{H} \mathbf{H}_{d r}$, respectively.

In the MIMO AF relaying systems, the instantaneous CSI of the first hop is often assumed to be available at the relay, thus the gain $G$ is given by [18]

$$
G^{2}=\frac{P_{r}}{P_{s} \lambda_{r s, 1}+\sum_{i=1}^{N} P_{i}\left|\mathbf{u}_{r s, 1}^{H} \mathbf{h}_{i}\right|^{2}+\sigma_{r}^{2}}
$$

and the output SINR in (4) can be further expressed as

$$
\gamma=\frac{\frac{P_{s}}{\sigma_{r}^{2}} \lambda_{r s, 1} \frac{P_{r}}{\sigma_{d}^{2}} \lambda_{d r, 1}}{\frac{P_{s}}{\sigma_{r}^{2}} \lambda_{r s, 1}+\left(\frac{P_{r}}{\sigma_{d}^{2}} \lambda_{d r, 1}+1\right)\left(\sum_{i=1}^{N} \frac{P_{i}}{\sigma_{r}^{2}} \varsigma_{i}+1\right)}=\frac{\alpha \beta}{\alpha+(\beta+1)(\tau+1)}
$$


where $\alpha=\gamma_{s} \lambda_{r s, 1}, \beta=\gamma_{r} \lambda_{d r, 1}, \tau=\sum_{i=1}^{N} \gamma_{i} \varsigma_{i}$ and $\varsigma_{i}=\left|\mathbf{u}_{r s, 1}^{H} \mathbf{h}_{i}\right|^{2}$ with $\gamma_{s}=P_{s} / \sigma_{r}^{2}, \gamma_{r}=P_{r} / \sigma_{d}^{2}, \gamma_{i}=P_{i} / \sigma_{r}^{2}$ being the SNRs of the source, relay and interference-to-noise ratio (INR) of the $i-$ th interferer.

Based on (6), we will derive the exact closed-form OP expression and asymptotic average SER of the MIMO $\mathrm{AF}$ transmission with variable-gain relaying protocol in the following sections.

\section{Outage Probability}

The OP of the relay network is defined as the probability that the end-to-end output SINR $\gamma$ drops below an acceptable threshold $\gamma_{t h}$, namely,

$$
P_{\text {out }}=\operatorname{Pr}\left(\gamma \leq \gamma_{t h}\right)=F_{\gamma}\left(\gamma_{t h}\right)
$$

where $F_{\gamma}(z)$ denotes the $\mathrm{CDF}$ of $\gamma$, which can then be calculated as

$$
\begin{aligned}
& F_{\gamma}(z)=\operatorname{Pr}\left\{\frac{\alpha \beta}{\alpha+(\beta+1)(\tau+1)}<z\right\} \\
& =\operatorname{Pr}\{(\beta-z) \alpha<z[(1+\beta)(1+\tau)]\} \\
& =\operatorname{Pr}\left\{\alpha>z \frac{\beta+1}{\beta-z}(\tau+1), \beta-z \leq 0\right\}+\operatorname{Pr}\left\{\alpha<z \frac{\beta+1}{\beta-z}(\tau+1), \beta-z>0\right\} \\
& =\operatorname{Pr}\{\beta \leq z\}+\operatorname{Pr}\left\{\alpha<z \frac{\beta+1}{\beta-z}(\tau+1), \beta-z>0\right\} \\
& =F_{\beta}(z)+\underbrace{\int_{I_{2}}^{\int_{0}^{\infty} F_{\alpha}\left[z \frac{x+1}{x-z} y\right] f_{\tau+1}(y) d y} f_{\beta}(x) d x}_{I_{1}}
\end{aligned}
$$

Theorem 1: The closed-form CDF expression of $\gamma$ can be derived as

$$
\begin{aligned}
& F_{\gamma}(z)=1-\sum_{p=1}^{N_{r s}} \sum_{q=\left|N_{r}-N_{s}\right|} \sum_{t=0}^{q} \sum_{r=1}^{w} \sum_{s=1}^{m} \sum_{j=0}^{m_{r}} \sum_{u=1}^{s-1} \sum_{v=\left|N_{d}-N_{r}\right|}^{N_{d r}\left(N_{d}+N_{r}\right) u-2 u^{2}} \sum_{k=0}^{t} \sum_{l=0}^{j+1} \sum_{n=0}^{v} C_{t}^{k} C_{j+1}^{l} C_{v}^{n} \frac{d_{u, v}}{v !}\left(\frac{u}{\gamma_{r}}\right)^{v+1} \frac{\delta_{r s}}{(s-1) ! \Omega_{r}^{s}} \\
& \times C_{s-1}^{j}(-1)^{s-j-1} \exp \left(\frac{1}{\Omega_{r}}\right) \frac{d_{p, q}}{t !}\left(\frac{p}{\gamma_{s}}\right)^{t}(t+j) !\left(\gamma_{s} \Omega_{r}\right)^{t+j+1} \frac{z^{t}}{\left(p z \Omega_{r}+\gamma_{s}\right)^{t+j+v+2}} \exp \left(\frac{u z}{\gamma_{r}} \frac{p \Omega_{r}-\gamma_{s}}{p z \Omega_{r}+\gamma_{s}}\right)\left[\gamma_{s}(z+1)\right]^{t-k} \\
& \times\left[-p z\left(1+\Omega_{r} z\right)\right]^{j-l+1}\left[-z\left(p \Omega_{r}-\gamma_{s}\right)\right]^{v-n} g\left(p z \Omega_{r}(z+1), \frac{u}{\gamma_{r}\left(p z \Omega_{r}+\gamma_{s}\right)}, k+l+n-t-j-1\right)
\end{aligned}
$$

where $g(\mu, \alpha, n)$ is given by

$$
g(\mu, \alpha, n)=\left\{\begin{array}{cl}
\exp (-\mu \alpha) \sum_{k=0}^{n} \frac{n !}{k !} \frac{\mu^{k}}{\alpha^{n-k+1}} & (n \geq 0) \\
\frac{(-1)^{n}}{\alpha^{n+1}} \frac{E i(-\mu \alpha)}{(-n-1) !}+\exp (-\mu \alpha) \mu^{n+1} \sum_{k=0}^{-n-2}(-\mu \alpha)^{k} \frac{(-n-1) !}{(-n-k-2) !} & (n<0)
\end{array}\right.
$$

and $E i(x)$ is the exponential integral function [22].

Proof: See Appendix A.

By replacing $z$ with $\gamma_{t h}$ in (9), the OP of the considered MIMO AF variable-gain relaying can be directly calculated.

Remark 1: A similar OP expression has been derived in [8, Eq. (12)] by neglecting the noise at the relay and assuming that each node has a single antenna only. For the scenario of multi-antenna relaying without interference, an analytical expression of outage probability has also been obtained in [4, Eq. (10)]. By considering that only 
one of the three nodes is equipped with multiple antennas and the relay is corrupted by only a single interferer, three formulas, namely, [18, Eq. (7)], [18, Eq. (17)] and [18, Eq. (31)] have been presented to calculate the OP of the variable-gain AF relaying. However, due to the existence of one-fold integral, the method in [18] requires a very complicated mathematical treatment to achieve the solution. On the contrary, here we derive the closed-form expression containing only elementary functions to efficiently evaluate the outage performance of a variable-gain AF relaying, where three nodes are each equipped with multiple antennas, the relay is impaired by multiple CCIs, and both the relay and destination are affected by the noise.

\section{Asymptotic Average SER}

To reveal the array gain and diversity order of the MIMO AF relaying, we will investigate the asymptotic behavior at high SNR in this section. The average SER of a wireless system with various modulation formats over AWGN channel is given by

$$
P_{s}=a E[Q(\sqrt{2 b \gamma})]=\frac{a}{2} \sqrt{\frac{b}{\pi}} \int_{0}^{\infty} \frac{\exp (-b u)}{\sqrt{u}} F_{\gamma}(u) d u
$$

where $a$ and $b$ are the parameters specified by the modulations. To derive the asymptotic average SER, we first express the upper bound of $\gamma$ as [21]

$$
\gamma=\frac{\alpha \beta}{\alpha+(\beta+1)(\tau+1)} \leq \min \left\{\frac{\alpha}{\tau+1}, \beta\right\}=\gamma_{u p}
$$

By letting $\varepsilon=\frac{\alpha}{\tau+1}$, the CDF of $\gamma_{u p}$ is given by

$$
F_{\gamma_{u p}}(z)=1-\left[1-F_{\varepsilon}(z)\right]\left[1-F_{\beta}(z)\right]=F_{\varepsilon}(z)+F_{\beta}(z)-F_{\varepsilon}(z) F_{\beta}(z)
$$

where the $\mathrm{CDF}$ of $\varepsilon$ can be denoted as

$$
F_{\varepsilon}(z)=\operatorname{Pr}\left\{\frac{\alpha}{\tau+1} \leq z\right\}=\operatorname{Pr}\{\alpha \leq z(\tau+1)\}=\int_{0}^{\infty} F_{\alpha}[z(x+1)] f_{\tau}(x) d x
$$

Due to the fact that the CDF of $\alpha$ can be approximated as [23]

$$
F_{\alpha}(x) \approx \frac{\Gamma_{N_{r s}}\left(N_{r s}\right)}{\Gamma_{N_{r s}}\left(N_{s}+N_{r}\right)}\left(\frac{x}{\gamma_{s}}\right)^{N_{s} N_{r}}
$$

where $N_{r s}=\min \left\{N_{s}, N_{r}\right\}, \Gamma_{n}(m)=\prod_{i=1}^{n} \Gamma(m-i+1)$ with $\Gamma(\cdot)$ being the Gamma function. Note that $\varsigma_{i}=$ $\left|\mathbf{u}_{r s, 1}^{H} \mathbf{h}_{i}\right|^{2}$ is a chi-square distributed random variable with two degrees of freedom and $1 / 2$ variance, the moment generating function (MGF) of $\tau=\sum_{i=1}^{N} \gamma_{i} \varsigma_{i}$ can be derived as

$$
\Psi_{\tau}(\xi)=\mathrm{E}_{\tau}[\exp (-\tau \xi)]=\prod_{i=1}^{N} \mathrm{E}_{\varsigma_{i}}\left[\exp \left(-\gamma_{i} \xi \varsigma_{i}\right)\right]=\int_{0}^{\infty} \exp \left(-\gamma_{i} \xi x\right) \exp (-x) d x=\prod_{i=1}^{N} \frac{1}{1+\gamma_{i} \xi}
$$

where $\Omega_{1}, \Omega_{2}, \ldots, \Omega_{w}$ as the distinct values of $\left\{\gamma_{i}\right\}_{i=1}^{N}$ with multiplicities $m_{1}, m_{2}, \ldots, m_{w}$, satisfying $\sum_{r=1}^{w} m_{r}=N$. By using partial fraction expansion, the MGF of $\tau$ can be rewritten as

$$
\Psi_{\tau}(\xi)=\sum_{r=1}^{w} \sum_{s=1}^{m_{r}} \frac{\delta_{r s}}{\left(1+\Omega_{r} \xi\right)^{s}}
$$


where the coefficient $\delta_{r s}$ is given by

$$
\delta_{r s}=\left.\frac{1}{\left(m_{r}-s\right) ! \Omega_{r}^{m_{r}-s}} \frac{\partial^{m_{r}-s}}{\partial_{\xi}^{m_{r}-s}}\left[\left(1+\Omega_{r} \xi\right)^{m_{r}} \Psi_{\tau}(\xi)\right]\right|_{\xi=-1 / \Omega_{r}}
$$

Finally, the PDF of $\tau$ can be calculated as

$$
f_{\tau}(x)=\sum_{r=1}^{w} \sum_{s=1}^{m_{r}} \frac{\delta_{r s}}{(s-1) ! \Omega_{r}^{s}} x^{s-1} \exp \left(-\frac{x}{\Omega_{r}}\right)
$$

Substituting (15) and (19) into (14), we have the approximate $F_{\varepsilon}(z)$ as

$$
\begin{aligned}
& F_{\varepsilon}(z) \approx \frac{\Gamma_{N_{r s}}\left(N_{r s}\right)}{\Gamma_{N_{r s}}\left(N_{s}+N_{r}\right)}\left(\frac{z}{\gamma_{s}}\right)^{N_{s} N_{r}} \sum_{r=1}^{w} \sum_{s=1}^{m_{r}} \frac{\delta_{r s}}{\Omega_{r}^{s}} \frac{1}{(s-1) !} \int_{0}^{\infty}(x+1)^{N_{s} N_{r}} x^{s-1} \exp \left(-\frac{x}{\Omega_{r}}\right) d x \\
& =\frac{\Gamma_{N_{r s}}\left(N_{r s}\right)}{\Gamma_{N_{r s}}\left(N_{s}+N_{r}\right)} \sum_{r=1}^{w} \sum_{s=1}^{m_{r}} \frac{\delta_{r s}}{\Omega_{r}^{s}} \Psi\left(s, N_{s} N_{r}+s+1 ; \frac{1}{\Omega_{r}}\right)\left(\frac{z}{\gamma_{s}}\right)^{N_{s} N_{r}}
\end{aligned}
$$

In deriving (20), we have used [22, Eq. (9.211.4)] to solve the integral term. Similarly, the CDF of $\beta$ can be approximated as [23]

$$
F_{\beta}(x) \approx \frac{\Gamma_{N_{d r}}\left(N_{d r}\right)}{\Gamma_{N_{d r}}\left(N_{r}+N_{d}\right)}\left(\frac{x}{\gamma_{r}}\right)^{N_{r} N_{d}}
$$

where $N_{d r}=\min \left\{N_{r}, N_{d}\right\}$, By taking (20) and (21) into (13), and let $\gamma_{r}=\kappa \gamma_{s}$, the asymptotic $F_{\gamma_{u p}}(z)$ in the high SNR regime, namely $\gamma_{s} \rightarrow \infty$, can be derived as

$$
F_{\gamma_{u p}}(z) \approx\left\{\begin{array}{cc}
\frac{\Gamma_{N_{r s}}\left(N_{r s}\right)}{\Gamma_{N_{r s}}\left(N_{s}+N_{r}\right)} \sum_{r=1}^{w} \sum_{s=1}^{m_{r}} \frac{\delta_{r s}}{\Omega_{r}^{s}} \Psi\left(s, N_{s} N_{r}+s+1 ; \frac{1}{\Omega_{r}}\right)\left(\frac{z}{\gamma_{s}}\right)^{N_{s} N_{r}} & N_{s}<N_{d} \\
\frac{\Gamma_{N_{r s}}\left(N_{r s}\right)}{\Gamma_{N_{r s}}\left(N_{s}+N_{r}\right)}\left[\sum_{r=1}^{w} \sum_{s=1}^{m_{r}} \frac{\delta_{r s}}{\Omega_{r}^{s}} \Psi\left(s, N_{s} N_{r}+s+1 ; \frac{1}{\Omega_{r}}\right)+\frac{1}{\kappa^{N_{s} N_{r}}}\right]\left(\frac{z}{\gamma_{s}}\right)^{N_{s} N_{r}} & N_{s}=N_{d} \\
\frac{\Gamma_{N_{d r}}\left(N_{d r}\right)}{\Gamma_{N_{d r}}\left(N_{r}+N_{d}\right) \kappa^{N_{r} N_{d}}}\left(\frac{x}{\gamma_{r}}\right)^{N_{r} N_{d}} & N_{s}>N_{d}
\end{array}\right.
$$

By substituting (22) into (11), the average SER at high SNR can be obtained as [24]

$$
P_{s}^{\infty} \approx\left(G_{a} \gamma_{s}\right)^{-G_{d}}
$$

where the diversity order $G_{d}$ and array gain $G_{a}$ can be, respectively, expressed as

$$
G_{a}=\left\{\begin{array}{cc}
G_{d}=\min \left\{N_{s}, N_{d}\right\} \cdot N_{r} & \\
{\left[\frac{a}{2} \sqrt{\frac{b}{\pi}} \frac{\Gamma_{N_{r s}}\left(N_{r s}\right)}{\Gamma_{N_{r s}}\left(N_{s}+N_{r}\right)} \sum_{r=1}^{w} \sum_{s=1}^{m_{r}} \frac{\delta_{r s}}{\Omega_{r}^{s}} \Psi\left(s, N_{s} N_{r}+s+1 ; \frac{1}{\Omega_{r}}\right) \frac{\Gamma\left(N_{s} N_{r}+1 / 2\right)}{b^{N_{s} N_{r}+1 / 2}}\right]^{-\frac{1}{N_{s} N_{r}}}} & N_{s}<N_{d} \\
{\left[\frac{a}{2} \sqrt{\frac{b}{\pi}} \frac{\Gamma_{N_{r s}\left(N_{r s}\right)}}{\Gamma_{N_{r s}}\left(N_{s}+N_{r}\right)}\left(\sum_{r=1}^{w} \sum_{s=1}^{m_{r}} \frac{\delta_{r s}}{\Omega_{r}^{s}} \Psi\left(s, N_{s} N_{r}+s+1 ; \frac{1}{\Omega_{r}}\right)+\frac{1}{\kappa^{N_{s} N_{r}}}\right) \frac{\Gamma\left(N_{s} N_{r}+1 / 2\right)}{b^{N_{s} N_{r}+1 / 2}}\right]^{-\frac{1}{N_{s} N_{r}}}} & N_{s}=N_{d} \\
{\left[\frac{a}{2} \sqrt{\frac{b}{\pi}} \frac{\Gamma_{N_{d r}}\left(N_{d r}\right)}{\Gamma_{N_{d r}}\left(N_{r}+N_{d}\right) \kappa^{N_{r} N_{d}}} \frac{\Gamma\left(N_{r} N_{d}+1 / 2\right)}{b^{N_{r} N_{d}+1 / 2}}\right]^{-\frac{1}{N_{r} N_{d}}}} & N_{s}>N_{d}
\end{array}\right.
$$

Remark 2: It is of interest to find that the existence of CCIs does not change the diversity order of the MIMO AF relaying systems. However, the CCIs can reduce the array gain of the relaying networks, thus deteriorate the system performance. 


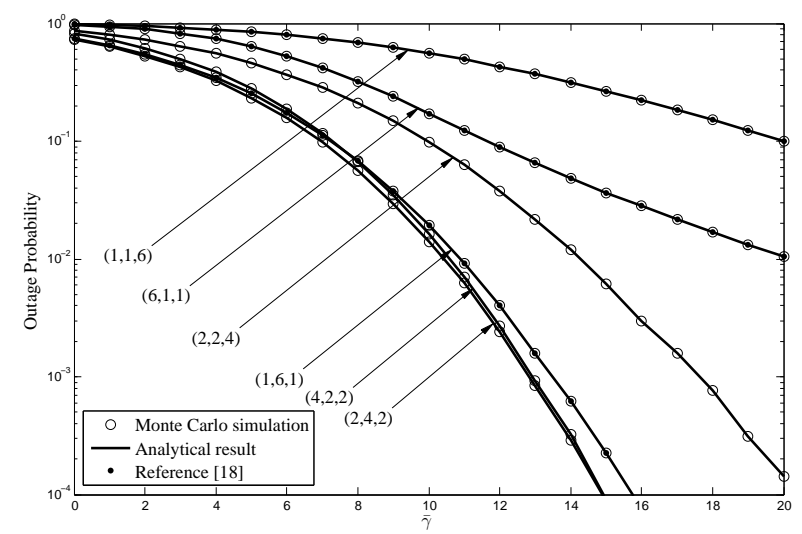

Fig. 1. OP of relaying networks with various antenna configurations

\section{Computer Simulation}

In this section, we carry out computer simulations to demonstrate the validity of the derived closed-form expressions, and investigate the effects of antenna configuration and power distribution among CCIs on the considered AF relay network. In all the plots, the label $\left(N_{s}, N_{r}, N_{d}\right)$ indicates the combination of the numbers of antennas at the source, relay and destination, $\gamma_{I}=\sum_{i=1}^{N} \gamma_{i}$ is the total INR of the CCIs.

Firstly, we examine the impact of antenna configuration on the MIMO AF relay network. The threshold is chosen as $\gamma_{t h}=0 \mathrm{~dB}$, and the relay is corrupted by one CCI with the INR being $\gamma_{I}=10 \mathrm{~dB}$. The OPs of fixed-gain relaying versus per hop SNR $\gamma_{s}=\gamma_{r}=\bar{\gamma}$ with six antenna combinations, namely, $(6,1,1),(1,6,1),(1,1,6),(4,2,2)$, $(2,4,2)$ and $(2,2,4)$, whose total antenna number is fixed as 8 , are plotted in Fig. 1. It is seen that the OP results through Monte Carlo experiments are highly consistent with those calculated from the theoretical formula in (9), implying that the derived closed-form expression accurately evaluates the OP performance of the MIMO AF relay networks. Furthermore, for $(6,1,1),(1,6,1)$ and $(1,1,6)$, the analytical results of our method also agree well with those through the theoretical formulas in [18], which confirm the correctness of our performance analysis again. Our formulae are formed by elementary functions which can be calculated efficiently, while the formulae in [18] are given in one-fold integral which requires complicated mathematical treatments to achieve the solution. Furthermore, we can see the OP of $(2,4,2)$ is better than that of $(4,2,2)$ and $(2,2,4)$. That is because each hop of $(2,4,2)$ has 8 links, while only the first hop of $(4,2,2)$ and the second hop of $(2,2,4)$ have 8 links, and thus $(2,4,2)$ can provide more diversity gain. In addition, the OP performance of $(6,1,1)$ and $(4,2,2)$ are better than that of $(1,1,6)$ and $(2,2,4)$, respectively. It could be inferred from this phenomenon that the $S-R$ hop has more impact on the performance of the system than the $R-D$ hop. This is due to CCIs existing at the receiver of the first hop, and thus more links should be deployed to the first hop to resist CCIs.

Secondly, we investigate the effect of CCI on the OP performance of the relay network. The antenna configurations are set to be $(2,2,4)$ and $(2,4,2)$, and the same simulation parameters as in the previous experiments are utilized. 


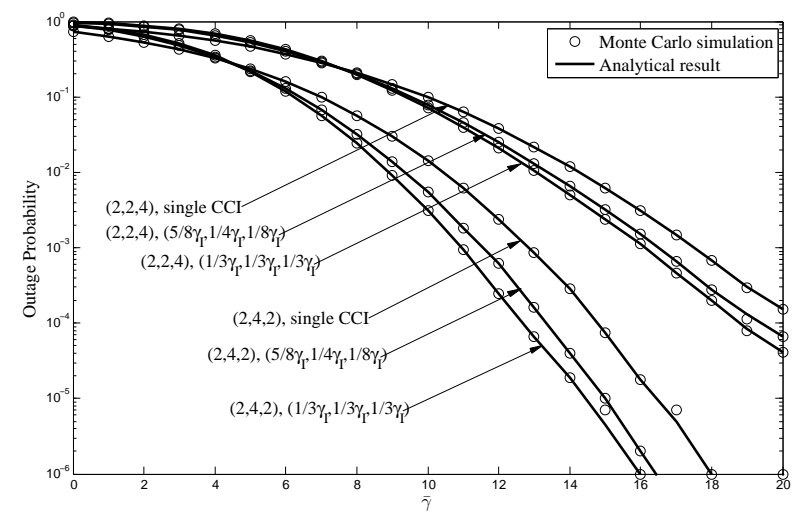

Fig. 2. OP of relaying networks with various scenarios of the CCIs

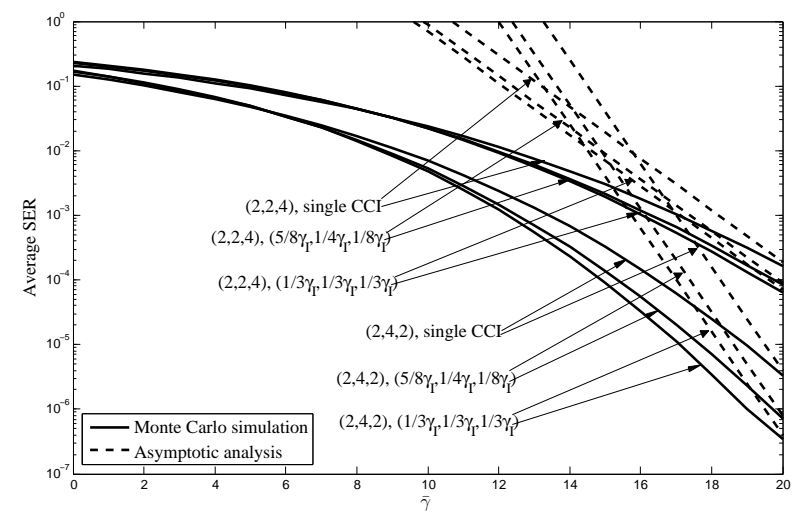

Fig. 3. Average SER of relaying networks with asymptotic analysis

Here various scenarios of the CCIs are considered. They are, scenario I: single CCI; scenario II: three CCIs with even power distribution as described by their INRs $\left(1 / 3 \gamma_{I}, 1 / 3 \gamma_{I}, 1 / 3 \gamma_{I}\right)$; scenario III: three CCIs with non-even power distribution as represented by three different INRs being $\left(5 / 8 \gamma_{I}, 1 / 4 \gamma_{I}, 1 / 8 \gamma_{I}\right)$. In Fig. 2 , we see that the relay network decays to the worst performance with single CCI. As the number of CCIs increases, we have better OP performance. We can infer from the phenomenon that constrained by the total power of CCIs, only one CCI is the best way to jam the communication of the relay network. We then study the impact of power distribution among the CCIs on the OP performance constrained by the same total INR. With the comparison of $\left(1 / 3 \gamma_{I}, 1 / 3 \gamma_{I}, 1 / 3 \gamma_{I}\right)$ and $\left(5 / 8 \gamma_{I}, 1 / 4 \gamma_{I}, 1 / 8 \gamma_{I}\right)$, it can be seen from the figure that the system with equal power distribution among the CCIs has the best OP performance due to the dispersing power of the CCIs.

Finally, the asymptotic average SER with various antenna configurations and CCIs are shown in Fig. 3. The fact that the curves of the asymptotic average SER approaches the Monte Carlo simulation results at high SNR demonstrate the validity of our asymptotic analysis. Meanwhile, We can see that the MIMO AF relay systems with 
the same antenna configuration have the same diversity order, but different array gain due to the different power distribution among CCIs. Moreover, it can be observed that the antenna configuration has significant effect on the considered relay network. For example, the performance of $(2,4,2)$ is better than that of $(2,2,4)$, because the diversity order of $(2,4,2)$ is 8 , while that of $(2,2,4)$ is only 4 .

\section{CONCLUSiON}

In this paper, we have studied the performance of a dual-hop MIMO AF relay network. We have considered that both transmit and receive BF are performed in each hop to steer the transmitted signal along the maximum eigenmode of the MIMO channel, and the relay is corrupted by multiple CCIs as well as noise. The exact closedform OP expression and asymptotic average SER for variable-gain relaying protocols have been derived. Computer simulations have been conducted to demonstrate the validity of the analytical results, enabling us to gain valuable insights into the effects of key parameters on the performance of the dual-hop AF relaying system, such as antenna configuration and power distribution among CCIs, as well as the benefit of implementing multiple antennas.

\section{APPENDIX A}

\section{PROOF OF THEOREM 1}

The CDF of $\alpha$, namely, $F_{\alpha}(x)$ is given by [25]

$$
F_{\alpha}(x)=1-\sum_{p=1}^{N_{r s}} \sum_{q=\left|N_{r}-N_{s}\right|}^{\left(N_{r}+N_{s}\right) p-2 p^{2}} d_{p, q} \exp \left(-\frac{p x}{\gamma_{s}}\right)\left(\sum_{t=0}^{q} \frac{p^{t}}{t !}\left(\frac{x}{\gamma_{s}}\right)^{t}\right)
$$

where the coefficient $d_{p, q}$ is dependent on the transmit-receive antenna combinations [25]. By using (19), the PDF $f_{\tau+1}(y)$ of $\tau+1$ is given by

$$
f_{\tau+1}(y)=f_{\tau}(y-1)=\sum_{r=1}^{w} \sum_{s=1}^{m_{r}} \sum_{j=0}^{s-1} \frac{\delta_{r s}}{(s-1) ! \Omega_{r}^{s}} C_{s-1}^{j}(-1)^{s-j-1} \exp \left(\frac{1}{\Omega_{r}}\right) y^{j} \exp \left(-\frac{y}{\Omega_{r}}\right)
$$

Thus $I_{1}$ in (8) can be rewritten as

$$
\begin{aligned}
& I_{1}=1-\sum_{p=1}^{N_{r s}} \sum_{q=\left|N_{r}-N_{s}\right|} \sum_{t=0}^{q} \sum_{r=1}^{w} \sum_{s=1}^{m_{r}} \sum_{j=0}^{s-1} \frac{\delta_{r s}}{(s-1) ! \Omega_{r}^{s}} C_{s-1}^{j}(-1)^{s-j-1} \exp \left(\frac{1}{\Omega_{r}}\right) \\
& \times \frac{d_{p, q}}{t !}\left(\frac{p}{\gamma_{s}}\right)^{t} z^{t}\left(\frac{x+1}{x-z}\right)^{t} \int_{0}^{\infty} y^{t+j} \exp \left[-\left(\frac{p z}{\gamma_{s}} \frac{x+1}{x-z}+\frac{1}{\Omega_{r}}\right) y\right] d y \\
& =1-\sum_{p=1}^{N_{r s}} \sum_{\left.q=\mid N_{r}+N_{s}\right) p-2 p^{2}}^{N_{s} \mid} \sum_{t=0}^{q} \sum_{r=1}^{w} \sum_{s=1}^{m_{r}} \sum_{j=0}^{s-1} \frac{\delta_{r s}}{(s-1) ! \Omega_{r}^{s}} C_{s-1}^{j}(-1)^{s-j-1} \exp \left(\frac{1}{\Omega_{r}}\right) \\
& \times \frac{d_{p, q}}{t !}\left(\frac{p}{\gamma_{s}}\right)^{t} z^{t}\left(\frac{x+1}{x-z}\right)^{t}(t+j) ! /\left(\frac{p z}{\gamma_{s}} \frac{x+1}{x-z}+\frac{1}{\Omega_{r}}\right)^{t+j+1}
\end{aligned}
$$

In deriving (28), we have applied [22, Eq. (3.351.3)]. Meanwhile, according to [25], we have the PDF of $\beta$ as

$$
f_{\beta}(x)=\sum_{u=1}^{N_{d r}} \sum_{v=\left|N_{d}-N_{r}\right|}^{\left(N_{d}+N_{r}\right) u-2 u^{2}} \frac{d_{u, v}}{v !}\left(\frac{u}{\gamma_{r}}\right)^{v+1} x^{v} \exp \left(-\frac{u}{\gamma_{r}} x\right)
$$


where the coefficient $d_{u, v}$ can be calculated in [25]. By substituting (28) and (29) into (8), it is not difficult to obtain $I_{2}$ in (8) as

$$
\begin{aligned}
& I_{2}=1-F_{\beta}(z)-\sum_{p=1}^{N_{r s}} \sum_{q=\left|N_{r}-N_{s}\right|}^{\left(N_{r}+N_{s}\right) p-2 p^{2}} \sum_{t=0}^{q} \sum_{r=1}^{w} \sum_{s=1}^{m_{r}} \sum_{j=0}^{s-1} \sum_{u=1}^{N_{d r}} \sum_{v=\left|N_{d}-N_{r}\right|} \sum^{\left.N_{d}+N_{r}\right) u-2 u^{2}} \frac{d_{u, v}}{v !}\left(\frac{u}{\gamma_{r}}\right)^{v+1} \frac{\delta_{r s}}{(s-1) ! \Omega_{r}^{s}} C_{s-1}^{j}(-1)^{s-j-1} \\
& \times \exp \left(\frac{1}{\Omega_{r}}\right) \frac{d_{p, q}}{t !}\left(\frac{p}{\gamma_{s}}\right)^{t}(t+j) ! z^{t} \underbrace{\int_{\gamma_{t h}}^{\infty} \frac{\left(\frac{x+1}{x-z}\right)^{t} x^{v}}{\left(\frac{p z}{\bar{\gamma}_{s}} \frac{x+1}{x-z}+\frac{1}{\Omega_{r}}\right)^{t+j+1}} \exp \left(-\frac{u}{\gamma_{r}} x\right) d x}_{I_{3}}
\end{aligned}
$$

With the change of variable $w=p z \Omega_{r}(x+1)+\gamma_{s}(x-z), I_{3}$ in (30) can be represented as

$$
\begin{aligned}
& I_{3}=\frac{\left(\gamma_{s} \Omega_{r}\right)^{t+j+1}}{\left(p z \Omega_{r}+\gamma_{s}\right)^{t+j+v+2}} \exp \left(\frac{u z}{\gamma_{r}} \frac{p \Omega_{r}-\gamma_{s}}{p z \Omega_{r}+\gamma_{s}}\right) \\
& \times \underbrace{\infty}_{I_{4}} \frac{\left[w+\gamma_{s}(z+1)\right]^{t}\left[z-p z\left(1+\Omega_{r} z\right)\right]^{j+1}\left[w-z\left(p \Omega_{r}-\gamma_{s}\right)\right]^{v}}{w^{t+j+1}} \exp \left(-\frac{u}{\gamma_{r}\left(p z \Omega_{r}+\gamma_{s}\right)} w\right) d w
\end{aligned}
$$

With the binomial expansion, $I_{4}$ can be written as

$$
\begin{aligned}
& I_{4}=\sum_{k=0}^{t} \sum_{l=0}^{j+1} \sum_{n=0}^{v} C_{t}^{k} C_{j+1}^{l} C_{v}^{n}\left[\gamma_{s}(z+1)\right]^{t-k}\left[-p z\left(1+\Omega_{r} z\right)\right]^{j-l+1}\left[-z\left(p \Omega_{r}-\gamma_{s}\right)\right]^{v-n} \\
& \times \underbrace{\int_{p z \Omega_{r}(z+1)}^{\infty} w^{k+l+n-t-j-1} \exp \left(-\frac{u}{\gamma_{r}\left(p z \Omega_{r}+\gamma_{s}\right)} w\right) d w}_{I_{5}}
\end{aligned}
$$

where the integral $g(\mu, \alpha, n)=\int_{\mu}^{\infty} x^{n} \exp (-\alpha x) d x$ can be calculated as (10), where the equations [22, Eq. (3.351.2)] [22, Eq. (3.351.4)] has been utilized. Thus, $I_{5}$ in (32) is given by

$$
I_{5}=g\left(p z \Omega_{r}(z+1), \frac{u}{\gamma_{r}\left(p z \Omega_{r}+\gamma_{s}\right)}, k+l+n-t-j-1\right)
$$

Now, substituting (33) into (32), substituting (32) into (31), substituting (31) into (30), and substituting (30) into (8), we finally obtain the closed-form CDF expression of $\gamma$ as (9).

\section{REFERENCES}

[1] A. J. Paulraj, D. A. Gore, R. U. Nabar, and H. Bolcskei, "An overview of MIMO communications - a key to gigabit wireless," Proceedings of the IEEE., vol. 92, no. 2, pp. 198-218, Feb. 2004.

[2] K. Loa, C.-C. Wu, S.-T. sheu, and el al., "IMT-advanced relay standards," IEEE Commun. Mag., vol. 48, no. 8, pp. 40-48, Aug. 2010.

[3] 3GPP TS36.912 V9.1.0, "Feasibility study for future advancement for E-UTRA (LTE-Advanced)," 2010.

[4] M. Li, M. Lin, Q. Yu, W.-P. Zhu and L. Dong, "Optimal Beamformer Design for Dual-Hop MIMO AF Relay Networks over Rayleigh Fading Channels," IEEE J. Sel. Areas Commun., vol. 30, no. 8, pp. 1402-1414, Sep. 2012.

[5] G. Amarasuriya, C. Tellambura, and M. Ardakani, "Performance analysis of hop-by-hop beamforming for dual-hop MIMO AF relay netorks," IEEE Trans. Commun., vol. 60, no. 7, pp. 1823-1837, July. 2012.

[6] C. Zhong, T. Ratnarajah, S. Jin, and K. K. Wong, "Performance Analysis of Optimal Single Stream Beamforming in MIMO Dual-Hop AF Systems," IEEE J. Sel. Areas Commun., vol. 30, no. 8, pp. 1415-1427, Sep. 2012. 
[7] C. Zhong, S. Jin, and K. K. Wong, "Dual-hop system with noisy relay and interference-limited destination," IEEE Trans. Commun., vol. 58, no. 3, pp. 764-768, Mar. 2010.

[8] H. A. Suraweera, H. K. Garg, and A. Nallanathan, "Performance analysis of two hop amplify-and-forward systems with interference at the relay," IEEE Commun. Lett., vol. 14, no. 8, pp. 692-694, Aug. 2010.

[9] H. A. Suraweera, D. S. Michalopoulos, R. S. Schober, G. K. Karagiannidis, and A. Nallanathan, "Fixed gain amplify-and-forward relaying with co-channel interference," in Proc. IEEE Int. Conf. Commun. (ICC), June. 2011, pp. 1-6.

[10] F. Al-Qahtani, T. Duong, C. Zhong, K. Qaraqe, and A. Alnuweiri, "Performance analysis of dual-hop AF systems with interference in Nakagami-m fading channels," IEEE Signal Process. Lett., vol. 18, no. 8, pp. 454-457, Aug. 2011.

[11] I. Trigui, S. Affes, and A. Stephenne, "On the ergodic capacity of amplify-and-forward relay channels with interference in Nakagami-m fading," IEEE Trans. Commun., vol. 61, no. 8, pp. 3136-3145, Aug. 2013.

[12] H. Ding, C. He, and L.-G. Jiang, "Performance analysis of fixed gain MIMO relay systems in the presence of co-channel interference," IEEE Commun. Lett., vol. 16, no. 7, pp. 1133-1136, Jul. 2012.

[13] H. Phan, T. Q. Duong, M. Elkashlan, and H.-J. Zepernick, "Beamforming amplify-and-forward relay networks with feedback delay and interference," IEEE Signal Process. Lett., vol.19, no.1, pp.16-19, Jan. 2012.

[14] W. Yang, Y. Cai and X. Xu, "Interference-limited MIMO relaying systems over Nakagami-m fading channels," Electron. Lett., vol. 48, no. 11, pp. 660-662, May. 2012.

[15] J. Wang, Y. Huang, C. Zhong, F. Al-Qahtani, Q. Wu and Y. Cheng, "Performance analysis of interference-limited dual-hop multiple antenna AF relaying systems with feedback delay," EURASIP Journal on Wireless Communications and Networking 2013., 2013: 284.

[16] Y. Huang, C. Li, C. Zhong, J. Wang and Q. Wu, "On the capacity of dual-hop multiple antenna AF relaying systems with feedback delay and CCI," IEEE Commun. Lett., vol. 17, no. 6, pp. 1200-1203, Jun. 2013.

[17] C. Zhong, H. A. Suraweera, and C. Yuen, "Outage probability analysis of dual-hop multiple antenna fixed-gain AF relay systems with interference", in Proc. IEEE WCNC, Apr. 2012, pp. 59-64.

[18] C. Zhong, H. A. Suraweera, A. Huang, Z. Zhang, and C. Yuen, "Outage probability of dual-hop multiple antenna AF relaying systems with interference," IEEE Trans. Commun., vol. 61, no. 1, pp. 108-119, Jan. 2013.

[19] G. Zhu, C. Zhong, H. A. Suraweera, Z. Zhang, and C. Yuen, "Outage probability of dual-hop multiple antenna AF systems with linear processing in the presence of co-channel interference," IEEE Trans. Wireless Commun., vol. 13, no. 4, pp. 2308-2321, Apr. 2014.

[20] G. Zhu, C. Zhong, H. Suraweera, Z. Zhang, C. Yuen, and R. Yin, "Ergodic capacity comparison of different relay precoding schemes in dual-hop AF systems with co-channel interference," IEEE Trans. Commun., vol. 62, no. 7, pp. 2314-2328, Jul. 2014.

[21] Y. Huang, F. Al-Qahtani, C. Zhong, Q. Wu, J. Wang, and H. Alnuweiri, "Performance analysis of multiuser multiple antenna relaying networks with co-channel interference and feedback delay," IEEE Trans. Commun., vol. 62, no. 1, pp. 59-73, Jan. 2014.

[22] I. S. Gradshteyn, I. M. Ryzhik, and A. Jeffrey, Table of integrals, series, and products, 6th ed. Academic press, 2000.

[23] S. Jin, M. R. Mckay, X.-Q. Gao, and I. B. Collings, "Asymptotic SER and outage probability of MIMO MRC in correlated fading," IEEE Signal Process. Lett., vol. 14, no. 1, pp. 9-11, Jan. 2007.

[24] Z. Wang, and G. B. Giannakis, "A simple and general parameterization quantifying performance in fading channels," IEEE Trans. Commun., vol. 51, no. 8, pp. 1389-1398, Aug. 2003.

[25] P. A. Dighe, R. K. Mallik, and S. S. Jamuar, "Analysis of transmit-receive diversity in Rayleigh fading," IEEE Trans. Commun., vol. 51, no. 4, pp. 694-703, Apr. 2003. 